\section{POS0606 DISEASE ACTIVITY AND PATIENTS-REPORTED OUTCOMES AFTER SWITCHING BETWEEN IL-6 RECEPTOR INHIBITORS AND JAK INHIBITORS: AN ANALYSIS FROM THE CORRONA REGISTRY}

A. Dua ${ }^{1}$, K. Ford ${ }^{2}$, S. Fiore ${ }^{3}$, D. A. Pappas ${ }^{4}$, J. Janak ${ }^{4}$, T. Blachley ${ }^{5}$, C. RobertsToler $^{5}$, K. Emeanuru ${ }^{6}$, J. Kremer ${ }^{7,8}$, A. Kivitz ${ }^{9} .{ }^{1}$ Northwestern University Feinberg School of Medicine, Department of Medicine, Division of Rheumatology, Chicago, IL, United States of America; ${ }^{2}$ Sanofi, Medical Affairs, Cambridge, MA, United States of America; ${ }^{3}$ Sanofi, Medical Affairs, Bridgewater, NJ, United States of America; ${ }^{4}$ Consortium of Rheumatology Researchers of North America (Corrona), Clinical Epidemiology, Waltham, MA, United States of America; ${ }^{5}$ Consortium of Rheumatology Researchers of North America (Corrona), Biostatistics, Waltham, MA, United States of America; ${ }^{6}$ Consortium of Rheumatology Researchers of North America (Corrona), Epidemiology, Waltham, MA, United States of America; ${ }^{7}$ Albany Medical College, Department of Medicine, Center for Rheumatology, Albany, NY, United States of America; ${ }^{8}$ Corrona LLC, Consortium of Rheumatology Researchers of North America (Corrona), Waltham, MA, United States of America; ${ }^{9}$ Altoona Center for Clinical Research, Altoona Arthritis \& Osteoporosis Center, Duncansville, PA, United States of America

Background: Rheumatoid arthritis (RA) patients who fail therapy may be switched to any of the five classes of biological disease-modifying antirheumatic drugs (DMARDs) and targeted synthetic DMARDs, to meet treatment goals. Physicians may hesitate to switch between Janus Kinase inhibitors (JAKi) and interleukin-6 receptor inhibitors (IL-6Ri) since they both impact IL-6 signalling and due to limited data on switching between the two classes.

Objectives: This retrospective, observational study based on the real-world Corrona RA registry aimed to describe the response in RA patients switching between IL-6Ri and JAKi.

Methods: Adult RA patients who initiated either IL-6Ri or JAKi after November 2012 and had a six-month post-initiation follow-up visit were eligible. Patients in 'Cohort A' initiated an IL-6Ri following discontinuation of a JAKi and those in 'Cohort B' initiated a JAKi following discontinuation of an IL-6Ri. Disease activity measures and patient-reported outcomes (PROs) were evaluated at baseline and at six-month follow-up. Within each group, change from baseline was assessed for Clinical Disease Activity Index (CDAI), Health Assessment Questionnaire (HAQ), pain, fatigue, tender joint count (TJC), swollen joint count (SJC), physician global assessment (MDGA), patient global assessment (PtGA) and morning stiffness duration. Proportion of patients achieving CDAl low disease activity (LDA), CDAI remission and minimal clinically important difference (MCID) for $\mathrm{HAQ}$, pain, fatigue, MDGA, PtGA were assessed. Adjusted linear and logistic regression models were performed for between-group comparisons (Cohort $A$ vs Cohort B) excluding initiators who switched therapy prior to six-month visit.

Results: Cohorts A and B included 122 and 144 initiators, respectively. Patients who switched to

IL-6Ri (vs JAKi) were younger (mean [SD] age, 56.2 [11.3] vs 58.9 [12.6] years), had higher baseline CDAI (23.2 [12.9] vs 20.2 [12.8]), had higher prior use of $\geq 2$ csDMARDs $(75 \%$ vs $65 \%)$, and were less likely to initiate therapy as monotherapy $(44 \%$ vs $50 \%)$.

In Cohort A, significant changes from baseline were observed for all continuous outcomes except $\mathrm{HAQ}$ and fatigue. In Cohort $\mathrm{B}$, a significant improvement was observed only for patient-reported pain (Table 1).

Table 1. Unadjusted Within-Group Change from Baseline to Six Months, Mean $(95 \% \mathrm{Cl})$, $\mathrm{n}$

\begin{tabular}{lll}
\hline Outcomes & Cohort A, N $=122$ & Cohort B, N $=144$ \\
\hline CDAI & $-4.7(-7.6,-1.9), 109$ & $-2.4(-5.2,0.4), 116$ \\
HAQ & $-0.0(-0.1,0.1), 105$ & $-0.1(-0.1,0.0), 118$ \\
Patient-reported pain & $-8.2(-13.4,-3.0), 109$ & $-5.9(-11.5,-0.2), 120$ \\
Patient-reported fatigue & $-4.4(-9.0,0.2), 109$ & $-1.7(-6.6,3.3), 117$ \\
TJC & $-1.6(-3.0,-0.1), 112$ & $-1.2(-2.6,0.3), 117$ \\
SJC & $-1.5(-2.5,-0.4), 112$ & $-0.4(-1.3,0.6), 117$ \\
MDGA & $-10.9(-15.6,-6.3), 112$ & $-4.3(-8.7,0.2), 117$ \\
PtGA & $-6.0(-11.2,-0.8), 109$ & $-4.8(-10.5,0.8), 120$ \\
Morning stiffness duration $^{\text {a }}$ & $-1.3(-2.2,-0.5), 109$ & $-0.1(-1.1,0.8), 118$ \\
\hline
\end{tabular}

${ }^{\mathrm{a}}$ Among those reporting morning stiffness at baseline.
In the adjusted between-group comparison (data not shown) of change from baseline, there were no significant differences in clinical outcomes between Cohorts $\mathrm{A}$ and $\mathrm{B}$.

In both cohorts, patients achieved CDAI LDA, CDAI remission, and MCIDs across other PROs (Figure 1). In the adjusted between-group comparison (data not shown), the results were similar with the exception of achievement of CDAI LDA among patients with moderate to high disease activity at baseline.
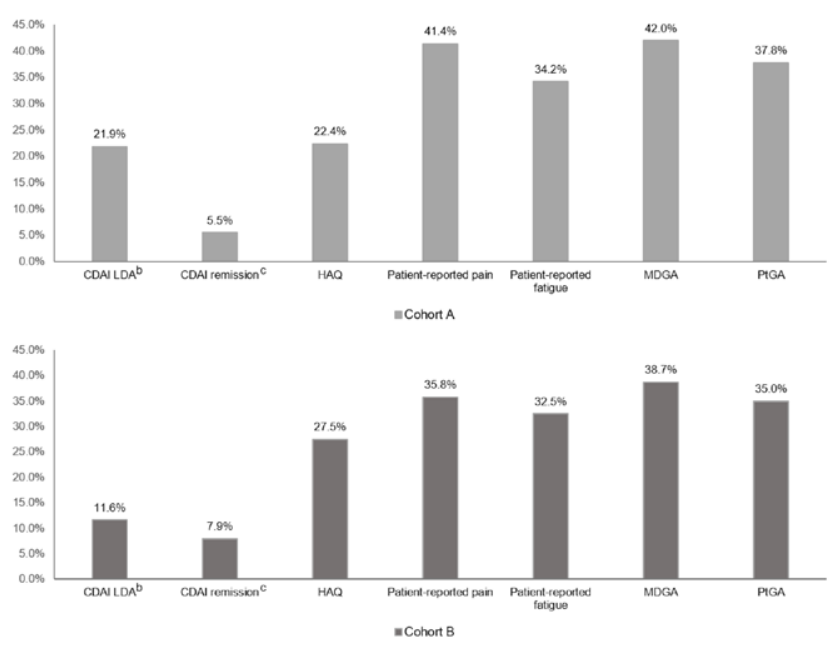

aCDAI LDA: >2.8 to $\$ 10$ units; CDAl remission: $\$ 2.8$ units; MCID for PROs were assessed, MCID for HAQ: 20.3 units; MCID for VAS of pain, fatigue, MDGA, and PIGA: 210 units. ${ }^{\circ}$ Among those in moderate or high disease activity at baseline. 'Among those in low,

Figure 1. Rates of CDAI LDA, CDAI Remission, and MCID for PROs ${ }^{a}$ at Six Months

Conclusion: In general, in both cohorts a substantial proportion of patients achieved CDAI LDA and MCID across PROs. Despite some overlap of JAKi and IL-6Ri therapies' on the IL-6 pathway, there are some distinct mechanisms of action which may result in meaningful improvements for a subset of patients.

Acknowledgements: Amy Praestgaard (Sanofi) contributed to the interpretation of the statistical analysis for this abstract. Medical writing support for this abstract was provided by Nupur Chaubey (Sanofi).

Disclosure of Interests: Anisha Dua Speakers bureau: AbbVie, Consultant of: Consulting/advisory board for AbbVie, Novartis, and Chemocentryx, Employee of: Board member of Vasculitis foundation and Chicago Rheumatism Society, Kerri Ford Shareholder of: Sanofi, Employee of: Sanofi, Stefano Fiore Shareholder of: Sanofi, Employee of: Sanofi. In addition, Stefano Fiore has a patent EP 19306553.9 USPTO \#s 62/799,698; 62/851,474; 62/935,395 issued, Dimitrios A Pappas Shareholder of: Corrona LLC, Consultant of: Sanofi, AbbVie, Gtech, Roche Hellas, and Novartis, Employee of: Corrona LLC. Board of directors, Corrona Research Foundation, Judson Janak: None declared, Taylor Blachley: None declared, Carla Roberts-Toler: None declared, Kelechi Emeanuru: None declared, Joel Kremer Consultant of: AbbVie Lilly, Novartis, Pfizer, BMS, Genentech, Regeneron, Sanofi, and Corrona, Grant/research support from: AbbVie, Lilly, Novartis, and Pfizer Alan Kivitz Shareholder of: Pfizer, Sanofi, GlaxoSmithKline, Gilead Sciences, Inc., and Novartis, Speakers bureau: Celgene, Merck, Lilly, Novartis, Pfizer, Sanofi, Flexion, and AbbVie, Consultant of: AbbVie, Boehringer Ingelheim, Flexion, Janssen, Pfizer, Sanofi, Regeneron, SUN Pharma Advanced Research, Gilead Sciences, Inc. In addition, Alan Kivitz reports other from Altoona Center for Clinical Research, PC, during the conduct of the study.

DOI: 10.1136/annrheumdis-2021-eular.1298 